\title{
Adult-onset systemic autoinflammatory disorders: a clinical approach
}

\author{
T. Borges ${ }^{1}$, A. Barbosa ${ }^{2}$, S. Silva ${ }^{1}$ \\ 'Department of Internal Medicine, Trofa Saúde Hospital Privado de Gaia, Vila Nova de Gaia, Portugal; \\ ${ }^{2}$ Department of Internal Medicine, Centro Hospitalar de São João, Porto, Portugal
}

\begin{abstract}
SUMMARY
Autoinflammatory disorders (AIDs) are a subgroup of immune-mediated syndromes that result from a primary dysfunction of the innate immune system. AIDs can be either monogenic or polygenic diseases. Unlike organspecific AIDs, systemic AIDs are characterized by fever and/or elevation of acute-phase reactants. This review aims to describe the most common adult-onset systemic AIDs, focusing mostly on polygenic and mixed-pattern diseases which are expected to be more prevalent in adult patients than monogenic AIDs overall. The literature was searched in Medline database. Organ-specific or childhood-onset systemic AIDs were excluded. AIDs were divided in three distinct groups: mixed-pattern, polygenic and adult-onset monogenic AIDs. Most adult-onset AIDs are polygenic but late-onset disease is not rare among monogenic AIDs such as familial Mediterranean fever (FMF). The diagnosis of systemic AIDs in adults is often delayed due to several factors and sometimes it is only established when amyloidosis or other complications are present. Therefore, it probably makes sense to primarily exclude common AIDs in adult patients with fever of unknown origin (and probably different presentations such as polyserositis) since a high prevalence of adult-onset Still's disease or FMF is usually expected. Colchicine, nonsteroidal anti-inflammatory drugs, steroids, immunosuppressive agents, interleukin-1 inhibitors and tumor necrosis factor antagonists constitute common therapeutic options for systemic AIDs.
\end{abstract}

Key words: Autoinflammatory disorders; innate immunity; adult.

\section{INTRODUCTION}

T mmune-mediated disorders (IMDs) include autoimmune diseases (ADs), allergies, immunodeficiencies and autoinflammatory disorders (AIDs). IMDs usually manifest during childhood but there are several exceptions, particularly in the first two subgroups. Instead, the majority of AIDs and immunodeficiencies typically manifest during childhood. AIDs result from a primary dysfunction of the innate immune system that can be determined by genetic mechanisms and/or exogenous factors (1). After recognition of molecular patterns, inflammasomes are assembled, resulting in clinical signs and/or laboratory evidence of inflammation (2). Hereditary periodic fever syndromes, such as familial Mediterranean fever (FMF), constitute prototypes of autoinflammation and are characterized by unprovoked recurrent febrile episodes of variable duration with intercalated periods of general well-being $(3,4)$. Rash, serositis, arthritis, aphthosis, gastrointestinal and ocular involvement may occur $(2,5)$. Meanwhile, organ-specific AIDs are characterized by elevation of inflammatory cytokines without inflammatory signs and/or elevation of acute-phase reactants. ADs such as systemic lupus erythematosus are primarily polygenic (geneticallycomplex) diseases involving both adaptive and innate immune systems (Table I) (6, $7)$. AIDs are primarily monogenic (recessive or dominant) and caused by mutations in genes involved in the activation or regulation of the inflammatory response, being expected to occur during the neonatal period or early infancy $(5,6,8)$. Exceptions include gout and pseudogout, Behçet's syndrome (BS), systemic-onset juvenile idiopathic arthritis (SJIA), spondyloarthritis (SpA), Crohn's disease, Schnitzler's
Corresponding author:

Tiago Borges

Rua Fernão de Magalhães 2 Fr E,

4404-501 Vila Nova de Gaia, Portugal

E-mail: mtiagoborges@gmail.com 
syndrome and periodic fever, aphthous stomatitis, pharyngitis and adenitis (PFAPA) syndrome, which are multifactorial syndromes $(6,9)$. These disorders often develop during adolescence or early adulthood and sometimes only manifest in the elderly $(6,9)$. Although the concept of autoinflammation was born after the description of monogenic hereditary periodic fever syndromes, dysfunctions in innate and adap-

Table I - Classification of autoimmune and autoinflammatory disorders according to their pattern of inheritance.

\begin{tabular}{|c|c|c|}
\hline & Autoinflammatory disorders & Autoimmune disorders \\
\hline 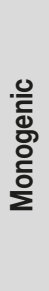 & $\begin{array}{l}\text { Familial Mediterranean fever } \\
\text { Tumor necrosis factor-associated periodic syndrome } \\
\text { Cryopyrin-associated periodic syndromes } \\
\text { Hypergammaglobulinemia D syndrome } \\
\text { PAPA syndrome } \\
\text { Majeed syndrome } \\
\text { Deficiency of interleukin-1 receptor antagonist } \\
\text { Blau syndrome }\end{array}$ & $\begin{array}{l}\text { Autoimmune polyendocrinopathy syndrome } \\
\text { Immune dysregulation polyendocrinopathy } \\
\text { entheropathy X-linked syndrome } \\
\text { Autoimmune lymphoproliferative syndrome } \\
\text { C1q deficiency }\end{array}$ \\
\hline 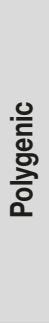 & $\begin{array}{l}\text { Gout } \\
\text { Pseudogout } \\
\text { Behçet's syndrome } \\
\text { Spondyloarthropathies } \\
\text { Crohn's disease } \\
\text { Still's disease } \\
\text { Schnitzler's syndrome } \\
\text { PFAPA syndrome }\end{array}$ & $\begin{array}{l}\text { Systemic lupus erythematosus } \\
\text { Rheumatoid arthritis } \\
\text { Sjögren syndrome } \\
\text { Systemic sclerosis } \\
\text { Inflammatory myopathies } \\
\text { Mixed connective tissue disease } \\
\text { Undifferentiated connective tissue disease } \\
\text { Systemic vasculitis } \\
\text { Overlap syndromes }\end{array}$ \\
\hline
\end{tabular}

PAPA - pyogenic arthritis, pyoderma gangrenosum, and acne syndrome; PFAPA - periodic fever, aphthous stomatitis, pharyngitis and adenitis syndrome.

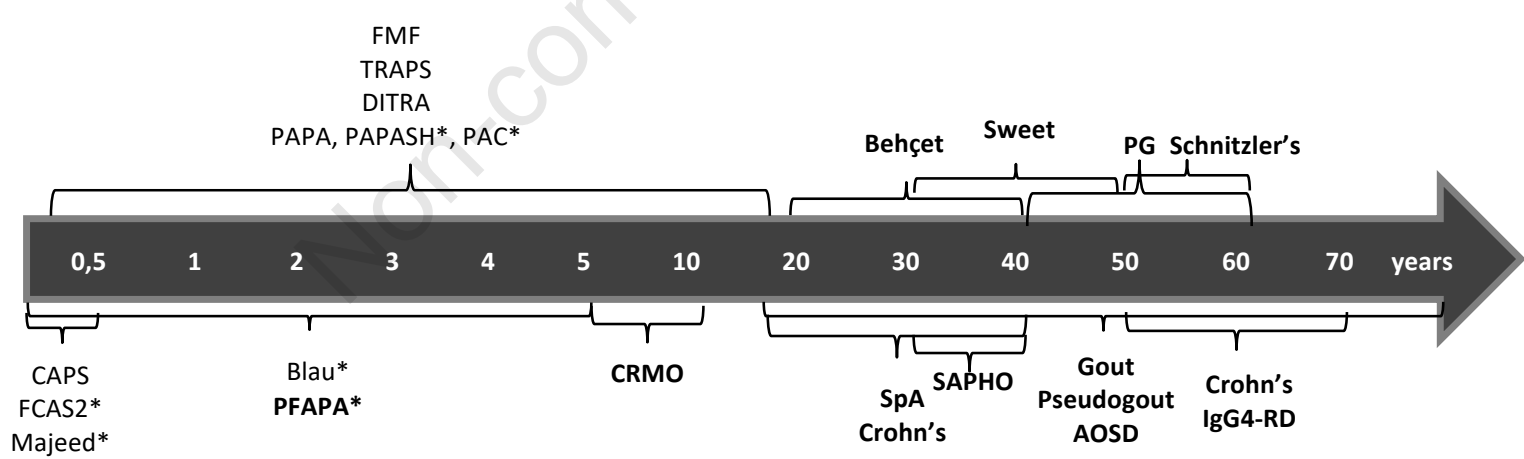

Figure 1 - Common age at presentation of adult-onset systemic autoinflammatory disorders (polygenic disorders are highlighted in bold and horizontal brackets represent an approximation of the most common age at presentation for each disorder). Other autoinflammatory disorders where the age at onset has a significant variability (e.g., PSORS2, aHUS) were not included.

AOSD - adult-onset Still's disease; CAPS - cryopyrin-associated periodic syndromes; CRMO - chronic recurrent multifocal osteomyelitis; DITRA - deficiency of IL-36 receptor antagonist; FCAS2 - NLRP12 associated fever syndrome; FMF - familial Mediterranean fever; IgG4-RD - immunoglobulin G4-related disease; PAC - pyoderma gangrenosum, acne, and ulcerative colitis syndrome; PAPA - pyogenic arthritis, pyoderma gangrenosum, and acne; PAPASH - pyogenic arthritis, pyoderma gangrenosum, acne and hidradenitis suppurativa syndrome; PFAPA - periodic fever, aphthous stomatitis, pharyngitis and adenitis syndrome; PG - pyoderma gangrenosum; SAPHO - synovitis, acne, pustulosis, hyperostosis, osteitis syndrome; SpA - spondyloarthritis; SS - Sweet syndrome; TRAPS - tumor necrosis factor-receptor associated periodic syndrome. *Anecdotal reports and/or unconfirmed cases of adult-onset disease. 
tive immune systems are not mutually exclusive and can occur even in monogenic AIDs (7).

Febrile episodes occur for the first time during infancy or childhood in almost $90 \%$ of the patients (10). About $10 \%$ only have clinical manifestations of AIDs after the age of 30 years and even inherited AIDs manifesting early in life are commonly diagnosed only during adulthood (see Figure 1 for common age at presentation of several AIDs) (11). The delay in diagnosis is common and may be partially explained by the relapsing-remitting pattern that characterizes many AIDs (12). Available treatments are usually successful and most of the patients survive until adulthood; chronic treatment is often required and therefore the clinical management of AIDs in adults also comprises such cases, aiming not only at the suppression of flares but also the prevention and treatment of complications such as amyloidosis. Surprisingly, fever of unknown origin (FUO) adult cohorts have demonstrated that the prevalence of adultonset Still's disease (AOSD) corresponds to about $5 \%$ of all cases (up to $15 \%$ in some cohorts), constituting sometimes the most common rheumatologic disorder, while FMF probably constitutes the second most prevalent AID (13). A study that included 266 adults with suspected AIDs identified 54 cases of NOD2-associated AIDs, 13 cases of FMF, 6 cases of tumor necrosis factor-receptor-associated periodic syndrome (TRAPS), 5 cases of cryopyrin-associated periodic syndromes (CAPS) and 1 case of hyperimmunoglobulinemia $\mathrm{D}$ with periodic fever syndrome (HIDS) (14).

The aim of this review is to describe adultonset systemic AIDs, focusing on the most prevalent diseases in this population. For this purpose, the literature was searched by combinations of keywords such as 'autoinflammatory', 'adult', 'innate immune system', 'fever', 'interleukin l' and 'systemic' in Medline database and articles published from 1980 to September 2018 were considered. Relevant references of the obtained articles were also taken in account. Those articles focusing on organ-specific AIDs or childhood-onset systemic AIDs were ex-
Table II - Classification of adult-onset systemic autoinflammatory disorders.

\begin{tabular}{|l|l|}
\hline & Examples \\
\hline \multirow{4}{*}{$\begin{array}{l}\text { Mixed-pattern } \\
\text { AIDs }\end{array}$} & Behçet's syndrome \\
& AOSD \\
& Spondyloarthritis?? \\
& lgG4-RD?? \\
\hline & Gout, Pseudogout \\
& Schnitzler's syndrome \\
Polygenic & PFAPA syndrome \\
AIDs & CRMO/SAPHO \\
& Crohn's disease \\
& Pyoderma gangrenosum, Sweet syndrome? \\
& Non-antibody associated vasculitis? \\
\hline Monogenic & FMF \\
AIDs & TRAPS \\
& CAPS \\
& (DITRA, aHUS, PSORS2, PAPA, PAPASH, PAC, FCAS2, \\
& Blau syndrome, Majeed syndrome)? \\
\hline
\end{tabular}

aHUS - atypical HUS; AOSD - adult-onset Still's disease; CAPS - cryopyrinassociated periodic syndromes; CRMO/SAPHO - chronic recurrent multifocal osteomyelitis/synovitis, acne, pustulosis, hyperostosis, osteitis syndrome; DITRA - deficiency of IL-36 receptor antagonist; FCAS2 - NLRP12 associated fever syndrome; FMF - familial Mediterranean fever; IgG4-RD - immunoglobulin G4-related disease; PAC - pyoderma gangrenosum, acne, and ulcerative colitis syndrome; PAPA - pyogenic arthritis, pyoderma gangrenosum, and acne syndrome; PAPASH - pyoderma gangrenosum, acne and hidradenitis suppurativa syndrome; PFAPA - periodic fever, aphthous stomatitis, pharyngitis and adenitis; PSORS2 - psoriasis susceptibility locus 2 syndrome; TRAPS - tumor necrosis factor-receptor associated periodic syndrome.

cluded. In this review, AIDs were divided in three subgroups: mixed-pattern, polygenic and adult-onset monogenic AIDs (Table II). In contrast to the first two subgroups, monogenic AIDs are not expected to manifest primarily in adults and therefore will be only briefly mentioned.

\section{MIXED-PATTERN AIDs}

The term mixed-pattern AIDs refers to the intersection between AIDs and ADs, sharing characteristics of both subgroups without clearly fitting into only one of them.

Systemic arthritis is characterized by arthritis and recurrent episodes of systemic inflammation and comprises both AOSD and SJIA $(7,15)$. HLA associations, pathogenesis and LACC1 mutations place it in the autoimmunoinflammatory spectrum (16). There is a bimodal age of distribution (15 to 25 years and 36 to 45 years), but late-onset disease can occur around 70 years of age (17). Patients typically present with arthralgia with or without arthritis, 
high-spiking fever, sore throat, evanescent salmon-pink rash, serositis, generalized lymphoid hyperplasia and/or hepatosplenomegaly $(7,18-20)$. Among other complications, macrophage activation syndrome is classically described $(18,20)$. Neutrophilic leukocytosis, thrombocytosis and elevated acute-phase reactants, liver abnormalities and hyperferritinemia are frequent, but glycosylated ferritin is more specific (7, 17, 21). Several classification criteria have been proposed, Yamaguchi's being the most used in clinical practice, whilst three phenotypes have been defined: monophasic, intermittent/polycyclic systemic and chronic articular (18-20). Systemic steroids and/or nonsteroidal anti-inflammatory drugs (NSAIDs) are the first therapeutic option, along with methotrexate or cyclosporine as steroid-sparing therapy $(17,18$, 20). Anakinra (particularly in systemic AOSD) or tocilizumab are preferred when biologics are considered, followed by TNF inhibitors when chronic arthritis is present (18-20).

BS is an inflammatory multisystemic disease (22). The International Chapel Hill Consensus Conference has described it as a variable vessel vasculitis (23). The relapsing-remitting pattern, the association with FMF, the description of MEFV as a susceptibility gene and other rare variants of innate immune system genes (NOD2, TLR4), the increased activity of neutrophils and the elevated levels of IL-1 $\beta$, the therapeutic response to colchicine and IL-1 inhibitors, and the absence of female predominance and autoantibodies favour an autoinflammatory origin $(2,4,22,24)$. However, other characteristics are concordant with an autoimmune origin: the strong association with major histocompatibility complex (MHC) class I (mainly HLA-B51), fever is not a prominent manifestation, the adaptive immune system and specifically $\mathrm{T}$ helper type 17 cells might have a role in the pathogenesis, T cell suppressing agents might induce a therapeutic response and childhood onset is uncommon $(22,24)$. BS typically manifests during early to middle adult life (4, $25)$. Recurrent attacks involving skin and/ or mucosa are almost universal and include oral and/or genital aphthous ulcers and papulopustular and/or acneiform skin lesions $(4,22)$. Pathergy and pustular skin lesions resemble pyogenic arthritis, pyoderma gangrenosum, and acne (PAPA) syndrome, whilst aphthous ulcers are similar to those seen in mevalonate kinase deficiency (4, 22). Ocular disease typically presents with panuveitis and retinal vasculitis (4). Vasculopathy predominantly affects veins and usually courses with deep vein thrombosis of the lower limbs (but not pulmonary embolism) and more rarely with Budd-Chiari and inferior vena cava syndromes and pulmonary artery aneurisms $(4,26)$. HughesStovin syndrome denotes the association between pulmonary artery aneurisms and deep vein thrombosis, while Mouth And Genital ulcers with Inflamed Cartilage (MAGIC) syndrome has been considered to be an intermediate condition between BS and relapsing polychondritis and/or cartilaginous inflammation as a consequence of systemic involvement $(4,27)$. Constitutional symptoms, musculoskeletal, cardiac, genitourinary, neurological (mainly headaches and parenchymal disease, but also neurovascular disease) and gastrointestinal (resembling Crohn's disease) involvement may also occur $(4,26)$. Two disease clusters have been described: the acne-arthritisenthesitis subtype and the vascular subset (26). The cutaneous pathergy reaction has a high specificity but lacks sensitivity $(4,26)$. There are no diagnostic tests but the International Study Group criteria are often used, while revised International Criteria for Behçet's disease might have a higher sensitivity and specificity (4). Treatment options include topical and systemic glucocorticoids, colchicine, cyclosporine, azathioprine, 5-ASA, cyclophosphamide, thalidomide, IFN $\alpha$, apremilast, anti-TNF antibodies (as second line treatment for most of the manifestations), embolization of pulmonary artery aneurisms, surgery, and possibly anticoagulants as adjuncts $(28,29)$. Colchicine is specifically indicated in erythema nodosum, papulopustular and acneiform skin lesions, acute arthritis and prevention of recurrent mucocutaneous lesions (28). 
Two syndromes should also be mentioned in the autoimmunoinflammatory spectrum of IMDs, even though their classification as AIDs is not straightforward. SpA includes four subtypes that are variably associated with HLA-B27 positivity: ankylosing spondylitis (AS), psoriatic arthritis (PA), reactive arthritis (RA) and enteropathic arthritis (EA) (30).

It cannot be considered a classic $\mathrm{AD}$ because it does not share female predominance, autoantibodies and response to $\mathrm{B}$ cell or T-cell target therapies with other ADs; moreover, the innate immune system appears to have an important role in its pathogenesis and some authors have included $\mathrm{SpA}$ in the autoinflammatory spectrum of IMDs $(21,31)$. Predominantly axial $\mathrm{SpA}$ includes AS (radiographic axial $\mathrm{SpA}$ ) and non-radiographic axial $\mathrm{SpA}$, while predominantly peripheral $\mathrm{SpA}$ includes the remaining subtypes $(32,33)$. Classification criteria have been developed for axial SpA (ASAS criteria) that include MRI findings and also for PA (CASPAR criteria) (34). NSAIDs are the first-line drug treatment, while sulfasalazine should be considered when peripheral arthritis is present and biologics when high disease activity is sustained despite conventional treatment $(33,35)$.

Immunoglobulin G4-related disease (IgG4-RD) has also been considered in the group of AIDs by some authors, but this is highly debatable. It is a multisystemic fibroinflammatory disorder characterized by elevated $\mathrm{IgG} 4$ levels and infiltration of IgG4-positive plasma cells $(36,37)$. T helper type 2 cells have an important role and drive the IgG4 subclass switch, but innate immunity might precede acquired immunity and induce storiform fibrosis through toll-like receptors (TLR)-mediated responses (38-40). Even though acute phase reactants may be elevated, IgG4-RD typically follows an insidious course without systemic signs of inflammation (36, 39). Prednisolone $(0.6 \mathrm{mg} / \mathrm{kg} /$ day for 2 to 4 weeks with gradual taper) is often the first option when symptoms are present or vital organs are affected, while rituximab has recently gained an important therapeutic role $(37,38)$.

\section{POLYGENIC AIDs}

Gout constitutes the most common inflammatory arthritis $(41,42)$. Its prevalence is about 3 to $6 \%$ in men and 1 to $2 \%$ in women, rising with advancing age (43). Other risk factors include ethnicity, specific mutations, obesity, hypertension, insulin resistance, menopause, comorbidities (e.g., renal dysfunction, heart failure, anemia, psoriasis, hematologic malignancies), lead exposure, drugs (e.g., diuretics) and consumption of beer, spirits, sugar-sweetened beverages, red meat and seafood $(41,43)$. The mean age at onset is about 49 years (44). Gout progresses in four stages: isolated hyperuricemia, crystal deposition, acute flares and advanced gout with tophi, chronic gouty arthritis and erosions (4143). Monosodium urate is the acute trigger of the NLRP3 inflammasome in acute gouty flares, recruiting caspase-1 which activates IL-1 $\beta(15,42,45,46)$. Acute gout is characterized by articular and periarticular inflammation with excruciating pain that commonly resolves after one or two weeks and typically involves the first metatarsophalangeal joints and, more rarely, ankles, knees, midfoot, wrists, elbow and bursae; fever can be present, particularly in polyarticular cases $(41,43)$. A presumptive diagnosis can be established on clinical grounds in typical cases like podagra but joint aspiration is necessary for exclusion of septic arthritis and/or definitive diagnosis (41). Acute phase reactants are often elevated; serum urate levels may be helpful but can be normal in acute gout (41, 43). Oral colchicine $(0.5 \mathrm{mg}$ two to three times per day), NSAIDs (particularly indomethacin and naproxen according to ACR guidelines) and/or steroids (and sometimes aspiration and intraarticular injection of steroids in monoarticular cases) constitute the mainstay of treatment in acute gout $(41,45,47)$. Colchicine or NSAIDs may also be used prophylactically $(43,45)$. Urate-lowering treatment (ULT) should be initiated one to two weeks after the acute attack has abated according to the European guidelines and continued if an acute at- 
tack develops (41). ULT includes xanthine oxidase inhibitors and uricosuric agents and is indicated in the presence of tophi or transplant-associated gout, renal dysfunction, urate nephrolithiasis, use of diuretics for heart failure or more than one attack of acute gout in 12 months (41). Doses should be up-titrated to reach serum urate levels $\leq 5-6 \mathrm{mg} / \mathrm{dL}(41,43)$. Other therapeutic options include recombinant uricase (pegloticase) and canakinumab (41-43, $45)$. Treatment of comorbidities and avoiding foods with high purine content is also important (41).

Calcium pyrophosphate deposition disease (CPPD) is caused by calcium pyrophosphate (CPP) crystals (48). It affects 4 to $7 \%$ of the adult population in developed countries (48). Risk factors include age, trauma, genetic factors, osteoarthritis (OA) and metabolic disturbances (e.g., hyperparathyroidism, hemochromatosis, hypomagnesemia, hypophosphatasia) (48). Besides sporadic and secondary cases, familial forms have been described (49). CPPD can be present in four patterns: asymptomatic CPPD with cartilage calcification (lanthanic variant), OA with CPPD, acute crystal arthritis (or pseudogout) and chronic inflammatory crystal arthritis (4850). In acute crystal arthritis, CPP crystals activate the NLPR3 inflammasome, possibly inducing fever and inflammatory signs that typically affect knees and wrists and may last for months (48). Definitive diagnosis is established by demonstrating positively birefringent rhomboid-shaped crystals in synovial fluid but diagnostic criteria have been proposed (Ryan and McCarty) $(48,50)$. Despite being a common finding, chondrocalcinosis is neither sensitive nor specific (50). Acute CPP crystal arthritis is treated with intraarticular steroids (often as first-line option), oral colchicine, NSAIDs and/or systemic steroids; IL-1 inhibitors have also been successful $(48,50)$.

Schnitzler's syndrome is a late-onset AID that invariably presents with both monoclonal gammopathy and chronic urticarial rash $(11,46,51,52)$. These features are often accompanied by fever, bone pain, arthritis, lymphadenopathy, hepatomeg- aly and/or splenomegaly, and leukocytosis (52). Weight loss, increased bone density and pancreatitis may occur $(21,15)$. Median age at presentation is 51 years and most of the patients are Caucasian with a male-female ratio of $1.5(11,46,53)$. It usually results from somatic mutations in NLRP3 $(9,51)$. The monoclonal protein appears to be the key for pathogenesis and IL-1 $\beta$ contributes to systemic inflammation $(11,52,54)$. Diagnostic criteria were proposed in 2001 (Lipsker et al.) and have been expanded to include the $\operatorname{IgG}$ variant; Strasbourg diagnostic criteria include chronic urticarial rash and monoclonal $\operatorname{IgM}$ or $\operatorname{IgG}$ as obligate criteria $(53,54)$. IgM kappa is the subtype in $85 \%$ (53). IL-1 antagonists are highly effective but do not decrease paraprotein levels and recurrence is common if stopped $(11,54)$. Steroids, rituximab, IFN $\alpha$, cyclosporine, colchicine, pefloxacin and thalidomide are moderately effective (53). Colchicine should be considered in patients without significant alterations in quality of life and without persistent elevation of acute phase reactants; anakinra should be considered if otherwise (54). Complications include hematologic malignancies in up to $20 \%$ (mainly Waldenström macroglobulinemia) and AA amyloidosis (11, 15, 46, 54).

PFAPA syndrome probably constitutes the most common periodic fever in children and it may be more common in males (11, $21,55-57)$. It has not a clear genetic basis and can be recognized in adults (mean age at onset of 20.9 years) with a higher rate of arthralgia and myalgia and a lower rate of aphthosis and chills $(8,56,58)$. Innate immune dysregulation results in the activation of adaptive immunity $(56,57)$. Unlike more recent updated criteria (e.g. Thomas et al.), original PFAPA criteria (Marshall, 1987) do not restrict the diagnosis to children, including the following items: recurrent fever with symptom-free intervals, normal growth and development, exclusion of cyclic neutropenia, and symptoms in the absence of upper respiratory infection with at least one additional clinical sign (aphthous stomatitis, cervical lymphadenitis and/or pharyngitis) (56). Fever typically 
lasts three to seven days and recurs every three to eight weeks; mild abdominal pain, leg pain, nausea and vomiting may occur $(56,57)$. Inflammatory markers are typically elevated only during flares $(11,56$, 57). Single dose steroids (e.g., prednisone $1-2 \mathrm{mg} / \mathrm{kg}$ ) are the mainstay of treatment and highly effective; NSAIDs appear more effective than acetaminophen, while IL-1 antagonists have induced prompt clinical benefits $(11,55-58)$. Colchicine can be used prophylactically and tonsillectomy in refractory cases $(56,58)$. Vitamin D supplementation (and possibly probiotic Streptococcus salivarus K12) may control flares (57). Complications are rare and many patients show no symptoms within a decade, although late relapses are common $(11,56,58)$.

Chronic non-infectious osteomyelitis characterizes bone AIDs, including chronic recurrent multifocal osteomyelitis (CRMO), synovitis, acne, pustulosis, hyperostosis, osteitis (SAPHO) syndrome, Majeed syndrome, deficiency of IL-1 receptor antagonist (DIRA), cherubism and juvenile mandibular chronic osteomyelitis $(59,60)$. CRMO primarily affects children and adolescents, but it may exclusively manifest during adulthood $(21,55,61)$. Inflammatory skin (e.g., severe acne, palmar plantar pustulosis, psoriasis vulgaris, generalized pustulosis and Sweet syndrome) and/ or intestinal (e.g., celiac disease, Crohn's disease, ulcerative colitis) lesions can also occur (62). SAPHO syndrome (anterior thoracic wall inflammatory syndrome or acquired hyperostosis syndrome) affects both children and adults, mostly females, but it is more common in the fourth and fifth decades of life, manifesting with hyperostosis and osteitis primarily involving the axial skeleton, erosive arthritis and possibly palmoplantar pustulosis, acne, hidradenitis suppurativa, enthesitis, sacroiliitis, uveitis, seizures and venous thrombosis (63-65). Diagnostic criteria have been proposed by Benhamou (1988) and Khan (2003) (63). In both syndromes, NSAIDs and analgesics are first-line therapeutic options, but steroids, methotrexate, antibiotics, isotretinoin, bisphosphonates and/or TNF inhibitors are used in refractory cases $(59,63,64)$. Human diseases involving chronic multifocal sterile osteomyelitis that are caused by single gene mutations will be mentioned below.

Neutrophilic dermatoses (ND) are characterized by sterile neutrophilic infiltrates and show a strong association with other AIDs $(66,67)$. The main clinical forms include pyoderma gangrenosum (PG), Sweet syndrome (SS, also known as acute febrile neutrophilic dermatosis), erythema elevatum diutinum (EED), subcorneal pustular dermatosis (SPD, also known as SneddonWilkinson disease) and overlap forms (67). Most of the cases are idiopathic but ND can be associated with hematologic malignancies, upper respiratory and gastrointestinal infections and AS (SS), inflammatory bowel disease and rheumatoid arthritis (PG) $(67,68)$. SS typically affects middle-aged women and may be categorized into classic, malignancy and drug-induced forms; it is a diagnosis of exclusion which requires the presence of two major criteria (abrupt onset of erythematous nodules or plaques plus histopathological evidence of neutrophilic infiltration with leukocytoclastic vasculitis) $(25,68,69)$. Lesions commonly appear on the face, upper trunk and/or proximal extremities, and inflammatory markers are typically elevated (69). PG is more common in females and its diagnosis requires a sterile skin biopsy (70). Both PG and SS are associated with the involvement of vital organs, but neurological involvement is restricted to SS (neuro-Sweet) (67). Systemic steroids constitute the therapeutic gold standard for SS and potassium iodide, colchicine and dapsone may be considered as steroid-sparing agents; NSAIDs, immunosuppressive agents, antibiotics, thalidomide and biologics (TNF and IL-1 antagonists) are used in refractory cases $(25,68)$. Wound care, local therapies and systemic steroids are primarily recommended for PG, whilst cyclosporine and TNF inhibitors may be considered thereafter (70). Crohn's disease is a granulomatous disorder $(51,71)$. Loss-of-function mutations in NOD2 (CARD15) act as risk factor (72). Inflammation is typically transmural and discontinuous along the gastrointes- 
tinal tract, commonly affecting the distal ileum, perianal area and colon $(21,72)$. Extraintestinal manifestations are common (72). It has a peak onset between 20 and 40 years of age (73). Recent insights about the pathogenesis of chronic granulomatous disorders (inflammasome activation, dysbalanced innate immune receptors) have placed it in the group of polygenic AIDs $(72,74)$. Steroids and immunosuppressive agents are first-line therapeutic options and TNF inhibitors are considered thereafter (21).

The evidence of vasculitis has been described in monogenic AIDs, including FMF, HIDS, CAPS, TRAPS, DIRA, STING-associated vasculopathy with onset in infancy (SAVI) and Blau syndrome (75). However, pro-inflammatory cytokines such as IL-1 are also increased in vasculitic disorders such as Kawasaki disease and nonantibody associated vasculitis, so vasculitis might be considered in the autoinflammatory spectrum of IMDs $(21,76)$.

\section{ADULT-ONSET MONOGENIC AIDS}

Monogenic AIDs have also been described in adults, but often such reports probably constitute delays in the diagnosis because long-standing symptoms are often overlooked. FMF and TRAPS probably represent an exception since their diagnosis in adults is not so rare.

In fact, FMF is the most common monogenic AID and results from mutations in the MEFV gene that encodes pyrin (marenostrin) $(9,55)$. Fever, serositis, erysipeloid erythema mainly affecting the lower limbs, acute scrotum attacks, monoarthritis of the large joints, myalgia and uveitis characterize the clinical picture $(71,77)$. Onset occurs before 20 years of age in more than $90 \%$ of patients and before 10 years of age in more than $50 \%(55,77)$. Late-onset disease has been described and, in a study of adult patients with FUO, about $80 \%$ had FMF as primary diagnosis (mean age was 27.8 years) $(8,78)$. This is associated with low-penetrance mutations and milder disease and may occur as late as 65 years (8).
TRAPS (familial Hibernian fever) is caused by autosomal dominant mutations in TNFRSF1A, coursing with fever, myalgia, nausea and vomiting, abdominal pain, serositis, arthritis, rash, lymphadenopathy, periorbital edema, conjunctivitis and/or scrotal pain $(11,21,71)$. Age at onset is variable but the mean is around seven years $(11,55)$. Late-onset disease is also related to low-penetrance mutations which carry a higher probability of milder and/or atypical manifestations and a lower risk of amyloidosis (8).

CAPS or cryopyrinopathies are usually caused by autosomal dominant gain-offunction mutations in NLRP3 and typically present with cold-induced urticarial rash with neutrophilic infiltration, including familial cold-associated syndrome (FCAS), Muckle-Wells syndrome (MWS) and neonatal-onset multisystem inflammatory disease/chronic infantile neurological cutaneous articular syndrome (NOMID/ CINCA) (79). Onset usually occurs during the neonatal period and early infancy, but adult-onset forms have been described; low-penetrance NLPR3 mutations seem to occur not only in these patients but also in adults with recurrent inflammatory attacks $(8,11,21,55)$.

Adult-onset disease has also been described as occurring in other monogenic AIDs such as PSTPIP1-related AIDs (e.g., PAPA, PAPASH or PAC syndromes), NLRP12 associated fever syndrome, deficiency of IL-36 receptor antagonist (DITRA), Majeed syndrome, PSORS2 syndrome and Blau syndrome; chronic multifocal sterile osteomyelitis caused by single gene mutations include PAPA (PSTPIP1 mutations), Majeed (LPIN2 mutations) and DIRA syndromes (IL1RN mutations) (80-88). These disorders will not be addressed here due to the rarity of diagnosis in adults.

\section{CONCLUSIONS}

Multifactorial polygenic disorders are expected to manifest later in life and, therefore, diseases like AOSD or gout are some of the most common AIDs in adults. However, late-onset disease can 
occur in monogenic AIDs such as FMF and NOD2-associated AIDs and less frequently in TRAPS or CAPS, often with low penetrance mutations $(8,14)$. Lowpenetrance mutations should be carefully interpreted to avoid overdiagnosis and overtreatment (see Table III for common therapeutic options). Perhaps, the demonstration of a high prevalence of AOSD, FMF and other AIDs in FUO adult cohorts makes it reasonable to primarily exclude such disorders and not only after malignancies or rheumatologic diseases such as ADs or vasculitides (and perhaps also when facing other clinical pictures such as polyserositis). Moreover, the diagnosis of AIDs in adults is often delayed, which can be explained by several factors, including the lack of symptom specificity, the unawareness of AIDs by clinicians (which may be more pronounced in adult patients because of the relative rarity of such syndromes and the higher likelihood of atypical forms in monogenic AIDs) or the relapsing-remitting pattern that is characterized by asymptomatic periods between flares. Probably, some examples that are presented in this review as adult-onset disease may constitute delays in the diagnosis. Sometimes this is only established when complications such as amyloidosis are present (89). Other AIDs invariably manifest during childhood and may persist lifelong. In fact, the clinical management of systemic AIDs in the adult population often includes AIDs occurring primarily in children, even though childhood-onset systemic AIDs were not the focus of this review.

In conclusion, systemic adult-onset AIDs are far more common than expected when monogenic disorders were first described. Since then, not only more monogenic AIDs have been added to the list and found to manifest later in life in some cases, but also the role of the innate immune system has been recognized as significant in the pathogenesis of some syndromes that are likely to be polygenic or mixedpattern AIDs. These should be born in mind when unexplained fever or elevated acute phase reactants are part of the clinical picture. Future studies should focus on clarifying the exact role of the innate immune system in the pathogenesis of such diseases (particularly SpA and IgG4-RD), developing classification criteria for a more accurate diagnosis, and distinguishing different phenotypes that might help individualize treatment.

Table III - Therapeutic options for adult-onset systemic autoinflammatory disorders.

\begin{tabular}{|c|c|c|c|c|c|c|}
\hline & Colchicine & NSAIDs & Steroids & IS & Anti-IL-1 & Anti-TNF $\alpha$ \\
\hline AOSD & 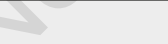 & 1 & 1 & 2 & 3 & 3 \\
\hline Behçet's syndrome & 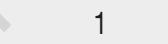 & & 1 & 2 & & 2 \\
\hline CAPS & & 2 & 2 & & 1 & \\
\hline CRMO/SAPHO & & 1 & 2 & 2 & 2 & 2 \\
\hline Crohn's disease & & & 1 & 2 & & 2 \\
\hline FMF & 1 & & & 3 & 2 & 3 \\
\hline Gout & 1 & 1 & 1 & & 2 & \\
\hline lgG4-related disease & & & 1 & 2 & & \\
\hline Neutrophilic dermatoses & 2 & 2 & 1 & 2 & & \\
\hline PFAPA & 2 & & 1 & & 2 & \\
\hline Pseudogout & 1 & 1 & 1 & & 2 & \\
\hline Schnitzler's syndrome & 2 & & 2 & & 1 & \\
\hline Spondyloarthritis & & 1 & 2 & 2 & & 2 \\
\hline TRAPS & & 1 & 1 & & 2 & 2 \\
\hline
\end{tabular}

1: first-line therapeutic option; 2: second-line therapeutic option; 3: third-line therapeutic option; Anti-IL-1 - interleukin-1 inhibitors; Anti-TNF $\alpha$ - tumor necrosis factor- $\alpha$ inhibitors; AOSD - adult-onset Still's disease; CAPS - cryopyrin-associated periodic syndromes; CRMO/SAPHO - chronic recurrent multifocal osteomyelitis/synovitis, acne, pustulosis, hyperostosis, osteitis syndrome; IS - immunosuppressive agents; FMF - familial Mediterranean fever; IgG4-RD - immunoglobulin G4-related disease; NSAIDs - nonsteroidal anti-inflammatory drugs; PFAPA - periodic fever, aphthous stomatitis, pharyngitis and adenitis syndrome; TRAPS - tumor necrosis factor-receptor associated periodic syndrome. 


\section{REFERENCES}

1. Grateau G, Hentgen V, Stojanovic KS, et al. How should we approach classification of autoinflammatory diseases? Nat Rev Rheumatol. 2013; 9: 624-9.

2. Azizi G, Khadem Azarian S, Nazeri S, et al. Monogenic Auto-inflammatory Syndromes: A Review of the Literature. Iran J Allergy Asthma Immunol. 2016; 15: 430-44.

3. van der Meer JW, Simon A. The challenge of autoinflammatory syndromes: with an emphasis on hyper-IgD syndrome. Rheumatology (Oxford). 2016; 55: ii23-9.

4. Ambrose NL, Haskard DO. Differential diagnosis and management of Behçet syndrome. Nat Rev Rheumatol. 2013; 9:79-89.

5. Zen M, Gatto M, Domeneghetti M, et al. Clinical guidelines and definitions of autoinflammatory diseases: contrasts and comparisons with autoimmunity-a comprehensive review. Clin Rev Allergy Immunol. 2013; 45: 227-35.

6. Doria A, Zen M, Bettio S, et al. Autoinflammation and autoimmunity: bridging the divide. Autoimmun Rev. 2012; 12: 22-30.

7. Ombrello MJ. Advances in the geneticallycomplex autoinflammatory diseases Semin Immunopathol. 2015; 37: 403-6.

8. Cantarini L, Lucherini OM, Rigante D. Caution should be used in the recognition of adultonset autoinflammatory disorders: facts or fiction? Front Immunol. 2013; 4: 96.

9. Ozen S, Bilginer Y. A clinical guide to autoinflammatory diseases: familial Mediterranean fever and next-of-kin. Nat Rev Rheumatol. 2014; 10: 135-47.

10. Kümmerle-Deschner JB. [Autoinflammatory Diseases as a Differential Diagnosis of Fever of Unknown Origin]. Dtsch Med Wochenschr. 2017; 142: 969-78.

11. Lachmann HJ. Autoinflammatory syndromes as causes of fever of unknown origin. Clin Med (Lond.). 2015; 15: 295-8.

12. González Garcia A, Patier de la Peña JL, Ortego Centeno N. Autoinflammatory diseases in adults. Clinical characteristics and prognostic implications. Rev Clin Esp. 2017; 217: 108-16.

13. Mulders-Manders CM, Simon A, BleekerRovers CP. Rheumatologic diseases as the cause of fever of unknown origin. Best Pract Res Clin Rheumatol. 2016; 30: 789-801.

14. Yao Q, Lacbawan F, Li J. Adult autoinflammatory disease frequency and our diagnostic experience in an adult autoinflammatory clinic. Semin Arthritis Rheum. 2016; 45: 633-7.

15. Cush JJ. Autoinflammatory syndromes. Dermatol Clin. 2013; 31: 471-80.

16. Aksentijevich I, McDermott MF. Lessons from characterization and treatment of the autoinflammatory syndromes. Curr Opin Rheumatol. 2017; 29: 187-94.
17. Mahroum N, Mahagna H, Amital H. Diagnosis and classification of adult Still's disease. J Autoimmun. 2014; 48-49: 34-7.

18. Kadavath S, Efthimiou P. Adult-onset Still's disease-pathogenesis, clinical manifestations, and new treatment options. Ann Med. 2015; 47: 6-14.

19. Gerfaud-Valentin M, Jamilloux Y, Iwaz J, Sève P. Adult-onset Still's disease. Autoimmun Rev. 2014; 13: 708-22.

20. Govoni M, Bortoluzzi A, Rossi D, Modena V. How I treat patients with adult onset Still's disease in clinical practice. Autoimmun Rev. 2017; 16: 1016-23.

21. Abramovits W, Oquendo M. Introduction to autoinflammatory syndromes and diseases. Dermatol Clin. 2013; 31: 363-85.

22. Gül A. Pathogenesis of Behçet's disease: autoinflammatory features and beyond. Semin Immunopathol. 2015; 37: 413-8.

23. Jenette JC, Falk RJ, Bacon PA, et al. 2012 revised International Chapel Hill Consensus Conference Nomenclature of Vasculitides. Arthritis Rheum. 2013; 65: 1-11.

24. Hedayaftar A. Behçet's Disease: Autoimmune or Autoinflammatory? J Ophtalmic Vis Res. 2013; 8: 291-3.

25. Nelson CA, Stephen S, Ashchyan HJ, et al. Neutrophilic dermatoses. Part I. Pathogenesis, Sweet syndrome, neutrophilic eccrine hidradenitis, and Behçet's disease. J Am Acad Dermatol. 2018; pii: S0190-9622(18)30504-8 [Epub ahead of print].

26. Yazici H, Seyahi E, Hatemi G, Yazici Y. Behçet syndrome: a contemporary view. Nat Rev Rheumatol. 2018; 14: 119.

27. Pak S, Logemann S, Dee C, Fershko A. Breaking the Magic: Mouth and Genital Ulcers with Inflamed Cartilage Syndrome. Cureus. 2017; 9: e1743.

28. Hatemi G, Christensen R, Bang D, et al. 2018 update of the EULAR recommendations for the management of Behçet's syndrome. Ann Rheum Dis. 2018; 77: 808-18.

29. Ozguler Y, Hatemi G. Management of Behçet's syndrome. Curr Opin Rheumatol. 2016; 28: 45-50.

30. Ritchlin CT, Colbert RA, Gladman DD. Psoriatic Arthritis. N Eng J Med. 2017; 376: 95770.

31. Ambarus C, Yeremenko N, Tak PP, Baeten D. Pathogenesis of spondyloarthritis: autoimmune or autoinflammatory? Curr Opin Rheumatol. 2012; 24: 351-8.

32. Schmitt SK. Reactive Arthritis. Infect Dis Clin North Am. 2017; 31: 265-77.

33. McAllister K, Goodson N, Warburton L, Rogers G. Spondyloarthritis: diagnosis and management: summary of NICE guidance. BMJ. 2017; 356: j839.

34. Akgul O, Ozgocmen S. Classification criteria 
for spondyloarthropathies. World J Orthop. 2011; 2: 107-15.

35. van der Heijde D, Ramiro S, Landewé R, et al. 2016 update of the ASAS-EULAR management recommendations for axial spondyloarthritis. Ann Rheum Dis. 2017; 76: 978-91.

36. Moriyama M, Nakamura S. Th1/Th2 Immune Balance and Other T Helper Subsets in IgG4Related Disease. Curr Top Microbiol Immunol. 2017; 401: 75-83.

37. Bozzalla Cassione E, Stone JH. IgG4-related disease. Curr Opin Rheumatol. 2017; 29: 223-7.

38. Della-Torre E, Lanzillotta M, Doglioni C. Immunology of IgG4-related disease. Clin Exp Immunol. 2015; 181: 191-206.

39. Umehara H, Nakajima A, Nakamura T, et al. IgG4-related disease and its pathogenesiscross-talk between innate and acquired immu nity. Int Immunol. 2014; 26: 585-95.

40. Watanabe T, Yamashita K, Kudo M. IgG4-Related Disease and Innate Immunity. Curr Top Microbiol Immunol. 2016; 401: 115-28.

41. Abhsishek A, Roddy E, Doherty M. Gout - a guide for the general and acute physicians. Clin Med (Lond). 2017; 17: 54-9.

42. So AK, Martinon F. Inflammation in gout: mechanisms and therapeutic targets. Nat Rev Rheumatol. 2017; 13: 639-47.

43. Dalbeth N, Merriman TR, Stamp LK. Gout. Lancet. 2016; 388: 2039-52.

44. Yu KH, Luo SF. Younger age of onset of gout in Taiwan. Rheumatology (Oxford). 2003; 42: 166-70.

45. Pascart T, Richette P. Current and future therapies for gout. Expert Opin Pharmacother. 2017; 18: 1201-11.

46. Sarrabay G, Grandemange S, Touitou I. Diagnosis of cryopyrin-associated periodic syndrome: challenges, recommendations and emerging concepts. Expert Rev Clin Immunol. 2015; 11: 827-35.

47. Shekelle PG, Newberry SJ, FitzGerald JD, et al. Management of Gout: A Systematic Review in Support of an American College of Physicians Clinical Practice Guideline. Ann Intern Med. 2017; 166: 37-51.

48. Rosenthal AK, Ryan LM. Calcium Pyrophosphate Deposition Disease. N Eng J Med. 2016; 374: 2575-84.

49. Ferrone C, Andracco R, Cimmino MA. Calcium pyrophosphate deposition disease: clinical manifestations. Reumatismo. 2012; 63: 246-52.

50. Rosenthal AK, Ryan LM. Crystal arthritis: Calcium pyrophosphate deposition - nothing "pseudo" about it! Nat Rev Rheumatol. 2011; 7: 257-8.

51. Manthiram K, Zhou Q, Aksentijevich I, Kastner DL. The monogenic autoinflammatory diseases define new pathways in human innate immunity and inflammation. Nat Immunol. 2017; 18: 832-42.

52. Pizzirani C, Falzoni S, Govoni M, et al. Dysfunctional inflammasome in Schnitzler's syndrome. Rheumatology (Oxford). 2009; 48: 1304-8.

53. de Koning HD. Schnitzler's syndrome: lessons from 281 cases. Clin Transl Allergy. 2014; 4: 41.

54. Simon A, Asli B, Braun-Falco M, et al. Schnitzler's syndrome: diagnosis, treatment, and follow-up. Allergy 2013; 68: 562-8.

55. Dueckers G, Sander O, Niehues T. Autoinflammatory diseases (AID). Klin Padiatr. 2014; 226: 133-42.

56. Wekell P, Karlsson A, Berg S, Fasth A. Review of autoinflammatory diseases, with a special focus on periodic fever, aphthous stomatitis, pharyngitis and cervical adenitis syndrome. Acta Paediatr. 2016; 105: 1140-51.

57. Rigante D, Gentileschi S, Vitale A, et al. Evolving Frontiers in the Treatment of Periodic Fever, Aphthous Stomatitis, Pharyngitis, Cervical Adenitis (PFAPA) Syndrome. Isr Med Assoc J. 2017; 19: 444-7.

58. Vigo G, Zulian F. Periodic fevers with aphthous stomatitis, pharyngitis, and adenitis (PFAPA). Autoimmun Rev. 2012; 12: 52-5.

59. Morbach H, Hedrich CM, Beer M, Girschick HJ. Autoinflammatory bone disorders. Clin Immunol. 2013; 147: 185-96.

60. Stern SM, Ferguson PJ. Autoinflammatory bone diseases. Rheum Dis Clin North Am. 2013; 39: 735-49.

61. Roderick MR, Shah R, Rogers V, et al. Chronic recurrent multifocal osteomyelitis (CRMO) - advancing the diagnosis. Pediatr Rheumatol Online J. 2016; 14: 47.

62. Sharma M, Ferguson PJ. Autoinflammatory bone disorders: update on immunologic abnormalities and clues about possible triggers. Curr Opin Rheumatol. 2013; 25: 658-64.

63. Carneiro S, Sampaio-Barros PD. SAPHO syndrome. Rheum Dis Clin North Am. 2013; 39: 401-18.

64. Greenwood S, Leone A, Cassar-Pullicino VN. SAPHO and Recurrent Multifocal Osteomyelitis. Radiol Clin North Am. 2017; 55: 1035-53.

65. Firinu D, Garcia-Larsen V, Manconi PE, Del Giacco SR. SAPHO Syndrome: Current Developments and Approaches to Clinical Treatment. Curr Rheumatol Rep. 2016; 18: 35.

66. Murthy AS, Leslie K. Autoinflammatory Skin Disease: A Review of Concepts and Applications to General Dermatology. Dermatology. 2016; 232: 534-40.

67. Prat L, Bouaziz JD, Wallach D, et al. Neutrophilic dermatoses as systemic diseases. Clin Dermatol. 2014; 32: 376-88.

68. Villarreal-Villarreal CD, Ocampo-Candiani J, Villarreal-Martínez A. Sweet Syndrome: 
A Review and Update. Actas Dermosifiliogr. 2016; 107: 369-78.

69. Paydas S. Sweet's syndrome: a revisit for hematologists and oncologists. Crit Rev Oncol Hematol. 2013; 86: 85-95.

70. Ashchyan HJ, Nelson CA, Stephen S, et al. Neutrophilic dermatoses. Part II. Pyoderma gangrenosum and other bowel and arthritis associated neutrophilic dermatoses. J Am Acad Dermatol. 2018; pii: S0190-9622(18)30503-6 [Epub ahead of print].

71. Verbsky JW. When to Suspect Autoinflammatory/Recurrent Fever Syndromes Pediatr Clin North Am. 2017; 64: 111-25.

72. Ciccarelli F, De Martinis M, Ginaldi L. An Update on Autoinflammatory Diseases. Curr Med Chem. 2013; 21: 261-9.

73. Uhlig HH. Monogenic diseases associated with intestinal inflammation: implications for the understanding of inflammatory bowel disease. Gut. 2013; 62: 1795-805.

74. Rieber N, Hector A, Kuijpers T, et al. Current concepts of hyperinflammation in chronic granulomatous disease. Clin Dev Immunol. 2012; 2012: 252460.

75. Ginsberg S, Rosner I, Rozenbaum M, et al. Autoinflammatory associated vasculitis. Semin Arthritis Rheum. 2016; 46: 367-71.

76. Peleg H, Ben-Chetrit E. Vasculitis in the autoinflammatory diseases. Curr Opin Rheumatol. 2017; 29: 4-11.

77. Berkun Y, Eisenstein EM. Diagnostic criteria of familial Mediterranean fever. Autoimmun Rev. 2014; 13: 388-90.

78. Kunimatsu J, Maeda J, Watanabe R, et al. Fever of unknown origin in the outpatient setting: A retrospective analysis of 30 cases of familial Mediterranean fever [Abstract]. Nihon Rinsho Meneki Gakkai Kaishi. 2016; 39: 130-9.

79. Goldbach-Mansky R. Current status of understanding the pathogenesis and management of patients with NOMID/CINCA. Curr Rheumatol Rep. 2011; 13: 123-31.
80. Marrakachi S, Guigue P, Renshaw BR, et al. Interleukin-36-Receptor Antagonist Deficiency and Generalized Pustular Psoriasis. N Engl J Med. 2011; 365: 620-8.

81. Jordan CT, Cao L, Roberson ED, et al. PSORS2 is due to mutations in CARD14. Am J Hum Genet. 2012; 90: 784-95.

82. Caorsi R, Marotto D, Insalaco A, et al. The phenotypic variability of PAPA syndrome: evidence from the Eurofever Registry. Pediatr Rheumatol Online J. 2015; 13: O8.

83. Ursani MA, Appleyard J, Whiteru O. Pyogenic Arthritis, Pyoderma Gangrenosum, Acne, Suppurative Hidradenitis (PA-PASH) Syndrome: An Atypical Presentation of a Rare Syndrome. Am J Case Rep. 2016; 17 : 587-91.

84. Zeeli T, Padalon-Brauch G, Ellenbogen E, et al. Pyoderma gangrenosum, acne and ulcerative colitis in a patient with a novel mutation in the PSTPIP1 gene. Clin Exp Dermatol. 2015; 40: 367-72.

85. Cox AJ, Zhao Y, Ferguson PJ. Chronic Recurrent Multifocal Osteomyelitis and Related Diseases-Update on Pathogenesis. Curr Rheumatol Rep. 2017; 19: 18.

86. Chen ES. Innate immunity in sarcoidosis pathobiology. Curr Opin Pulm Med. 2016; 22: 469-75.

87. Yao Q, Piliang M, Nicolacakis K, Arrossi A. Granulomatous pneumonitis associated with adult-onset Blau-like syndrome. Am J Resp Crit Care Med. 2012; 186: 465-6.

88. Hoffmann SR, Schnabel A, Rösen-Wolff A, et al. Chronic Nonbacterial Osteomyelitis: Pathophysiological Concepts and Current Treatment Strategies. J Rheumatol. 2016; 43: 1956-64.

89. Kluk J, Rustin M, Brogan PA, et al. Chronic atypical neutrophilic dermatosis with lipodystrophy and elevated temperature syndrome: a report of a novel mutation and review of the literature. Br J Dermatol. 2014; 170: 215-7. 